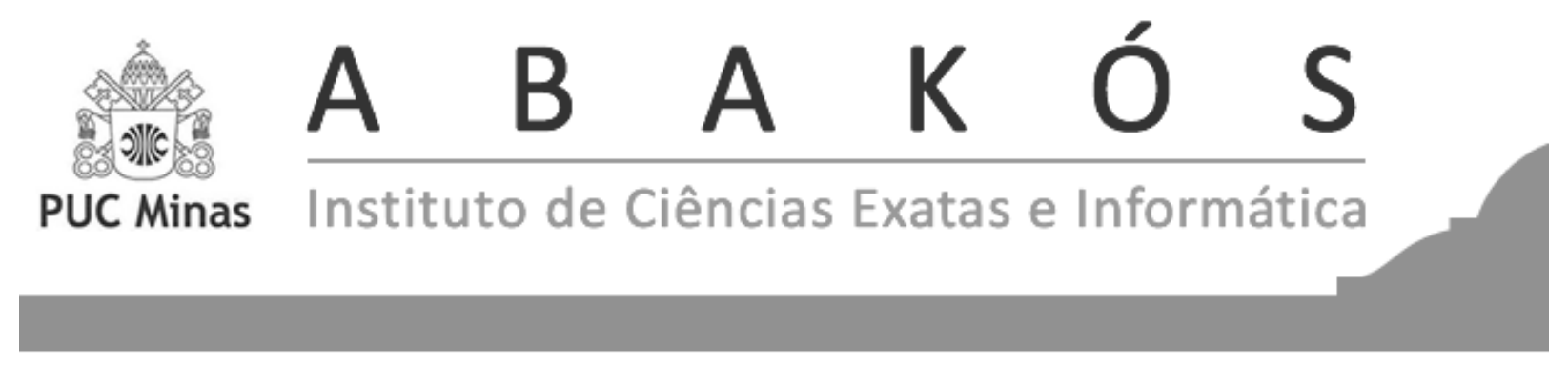

(c) (c) Licença Creative Commons Attribution 4.0 International

\title{
Um Novo Modelo para o Imposto de Renda Pessoa Física no Brasil* $^{*}$
}

A New Model for Personal Income Tax in Brazil

Thiago Linhares Brant Reis ${ }^{1}$ Alexandre Duarte Washington ${ }^{2}$

\begin{abstract}
Resumo
No Brasil, o Imposto de Renda Pessoa Física (IRPF) é calculado com base em cinco grandes faixas de renda. Neste trabalho é proposto um modelo mais equitativo, baseado em uma função contínua, utilizando-se técnicas de Cálculo Diferencial e Integral - uma aplicação de modelagem matemática à macroeconomia. Com o auxílio de um algoritmo (apresentado no apêndice), o método atual e o novo método são comparados, buscando-se evidenciar algumas vantagens do segundo. O novo modelo é relativamente simples, tornando-se atrativo para aplicação pelos gestores da Economia. Ademais, pretende-se também contribuir para a redução da sonegação fiscal.
\end{abstract}

Palavras-chave: Imposto de renda pessoa física, Cálculo Diferencial e Integral, Soma de Riemann, Macroeconomia.

${ }^{*}$ Submetido em 19/06/2018 - Aceito em 22/03/2019

${ }^{1}$ Mestre em Ensino de Matemática pelo Programa de Pós-Graduação em Ensino de Ciências e Matemática da PUC Minas , Brasil-adwtlbr@gmail.com

${ }^{2}$ Mestre em Matemática pelo Programa de Pós-Graduação em Matemática do Instituto de Ciências Exatas da UFMG , Brasil - adwtlbr@gmail.com 


\begin{abstract}
n Brazil, Personal Income Tax (IRPF , for the Portuguese acronym) is calculated based on five large income brackets. We propose a more equitable model, based on a continuous function, using Differential and Integral Calculus - an application of mathematical modeling to macroeconomics. With the aid of an algorithm (presented inthe appendix), the current method and the new method are compared, seeking to show some advantages of the second. The new model is relatively simple, becoming attractive for application by the managers of the Economy. In addition, it is also intended to contribute to the reduction of fiscal evasion.
\end{abstract}

Keywords: Individual income tax, Differential and Integral Calculus, Riemann Sum, Macroeconomics. 


\section{INTRODUÇÃO}

No Brasil, o Imposto de Renda Pessoa Física (abreviado IRPF) foi instituído através da Lei 4.625, em 31 de dezembro de 1922, durante o governo de Artur Bernardes, que orçou a Receita dos Estados Unidos do Brasil para o ano seguinte. Atualmente é regulamentado pela lei $\mathrm{n}^{\mathrm{o}} 13.149$, de 21 de julho de 2015 , a qual teve origem na medida provisória $\mathrm{n}^{\mathrm{o}} 670$, de $10 \mathrm{de}$ março de 2015.

Desde então, a correção da tabela do imposto de renda fica a cargo dos governos e os critérios ficam a cargo do poder executivo. Como a atualização da tabela do imposto de renda é pouco frequente, o governo consegue ampliar naturalmente sua arrecadação, pois os salários tendem a aumentar, acompanhando o índice da inflação, enquanto a base de cálculo do imposto permanece a mesma. Mesmo com uma tabela congelada, aumentos salariais abaixo da inflação podem fazer com que o contribuinte mude de faixa de tributação, e tenha sua carga tributária aumentada, disse Eduardo Cury (relator do projeto que trata da correção da tabela do IR na Comissão de Finanças e Tributação da Câmara dos Deputados), numa entrevista ao jornal Estadão em 18 de janeiro de 2018.

A proposta dos autores é criar um novo modelo para o IRPF no Brasil, mais equitativo, utilizando o Cálculo Diferencial e Integral.

\subsection{Problema Motivador}

Historicamente, a alíquota do IRPF é definida de acordo com "faixas" de renda das pessoas físicas. O grau de justiça desse sistema tem sido questionado, por diversos motivos. Um argumento é que essas faixas são poucas e largas. Além disso, a correção da tabela é realizada com baixa frequência. A estratificação pobre e a constante defasagem da tabela oneram desnecessariamente as pessoas físicas.

O problema, então, é o seguinte: como tornar o imposto de renda melhor distribuído em relação aos valores percebidos pelas pessoas físicas? Além disso, como fazer isso de forma compatível com o modelo em vigor?

A proposta deste artigo é resolver a questão trocando-se o modelo estratificado por um modelo suave, de maneira que pequenas mudanças na renda sejam refletidas no cálculo final do imposto. Como o operador de integral é um "restaurador de continuidade", foi escolhido como ferramenta principal. A proximidade do modelo proposto com o modelo atual, não obstante as vantagens do novo modelo, pode ser obtida através do ajuste adequado dos parâmetros da função integrando. 


\section{DESENVOLVIMENTO}

Durante a pesquisa, buscou-se conhecer um pouco sobre a história do IRPF no Brasil, analisar e interpretar graficamente a atual Tabela Progressiva Mensal do IRPF, criar um novo modelo para o cálculo desse IRPF. Implementou-se também, um algoritmo através da linguagem de programação $\mathrm{C}++$, com o objetivo de comparar os impostos cobrados pelo atual IRPF e pelo modelo proposto.

\subsection{A Tabela do IRPF}

A tabela em vigor do IRPF é representada na tabela 1.

Tabela 1 - Tabela Progressiva Mensal do IRPF.

\begin{tabular}{c|c|c}
\hline Base de Cálculo $(\mathrm{R} \$)$ & Alíquota $(\%)$ & Parcela a deduzir do IR (R\$) \\
\hline Até $1.903,98$ & - & - \\
\hline De $1.903,99$ até $2.826,65$ & 7,5 & 142,80 \\
\hline De $2.826,66$ até $3.751,05$ & 15 & 354,80 \\
\hline De 3.75106 até $4.664,68$ & 22,5 & 636,13 \\
\hline Acima de $4.664,68$ & 27,5 & 869,36 \\
\hline
\end{tabular}

Fonte: http://www.planalto.gov.br/ccivil_03/_ato2015-2018/2015/lei/113149.htm.

A Tabela 1 pode ser colocada em formato de gráfico conforme mostrado na Figura 1.

Figura 1 - Tabela Progressiva Mensal do IRPF.

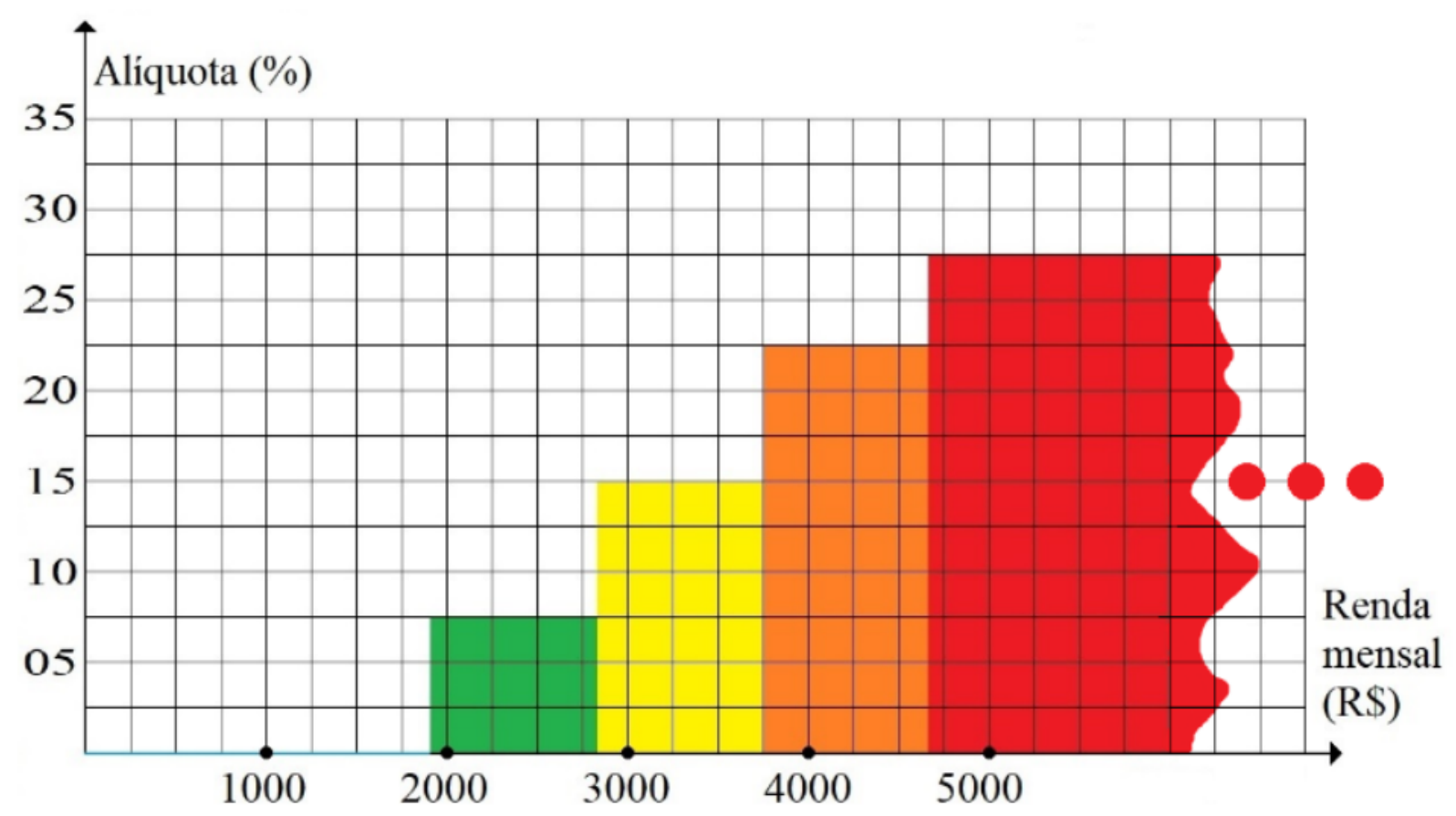

Fonte: elaborado pelos autores a partir da Tabela 1.

Suponhamos que uma pessoa receba um salário mensal bruto de $\mathrm{R} \$ 3.000,00$. O cálculo 
do imposto no modelo real se dá por:

- Alíquota: $15 \%$ de $\mathrm{R} \$ 3.000,00=\mathrm{R} \$ 450,00$.

- Parcela a deduzir: $\mathrm{R} \$ 354,80$.

- Imposto devido: $\mathrm{R} \$ 450,00-\mathrm{R} \$ 354,80=\mathrm{R} \$ 95,20$ mensais.

A conformação do gráfico sugere uma soma de Riemann. É um gráfico de barras, o qual denominaremos de modelo estratificado. Segue naturalmente a ideia: e se, em vez de um gráfico de barras, fosse utilizado um gráfico de função?

\subsection{Modelagem Suave}

O objetivo é encontrar uma função contínua suave $\mathrm{y}=\mathrm{f}(\mathrm{x})$ compatível com o gráfico anterior. Uma função contínua suave é uma função que possui derivadas de todas as ordens em todo o seu domínio. Essa função será aqui denominada de modelo suave. A partir de uma representação por somas de Riemann, deve-se determinar uma possível expressão da função. Tal função deve ter pelo menos as seguintes propriedades:

(1) possuir domínio igual aos reais não negativos;

(2) ser identicamente nula num trecho inicial;

(3) possuir exatamente um ponto de inflexão (oblíqua e crescente);

(4) possuir exatamente uma reta assíntota horizontal;

(5) ser suave.

O aspecto de tal função, superposta ao gráfico de barras, é mostrado na Figura 2. 
Figura 2 - Gráficos superpostos.

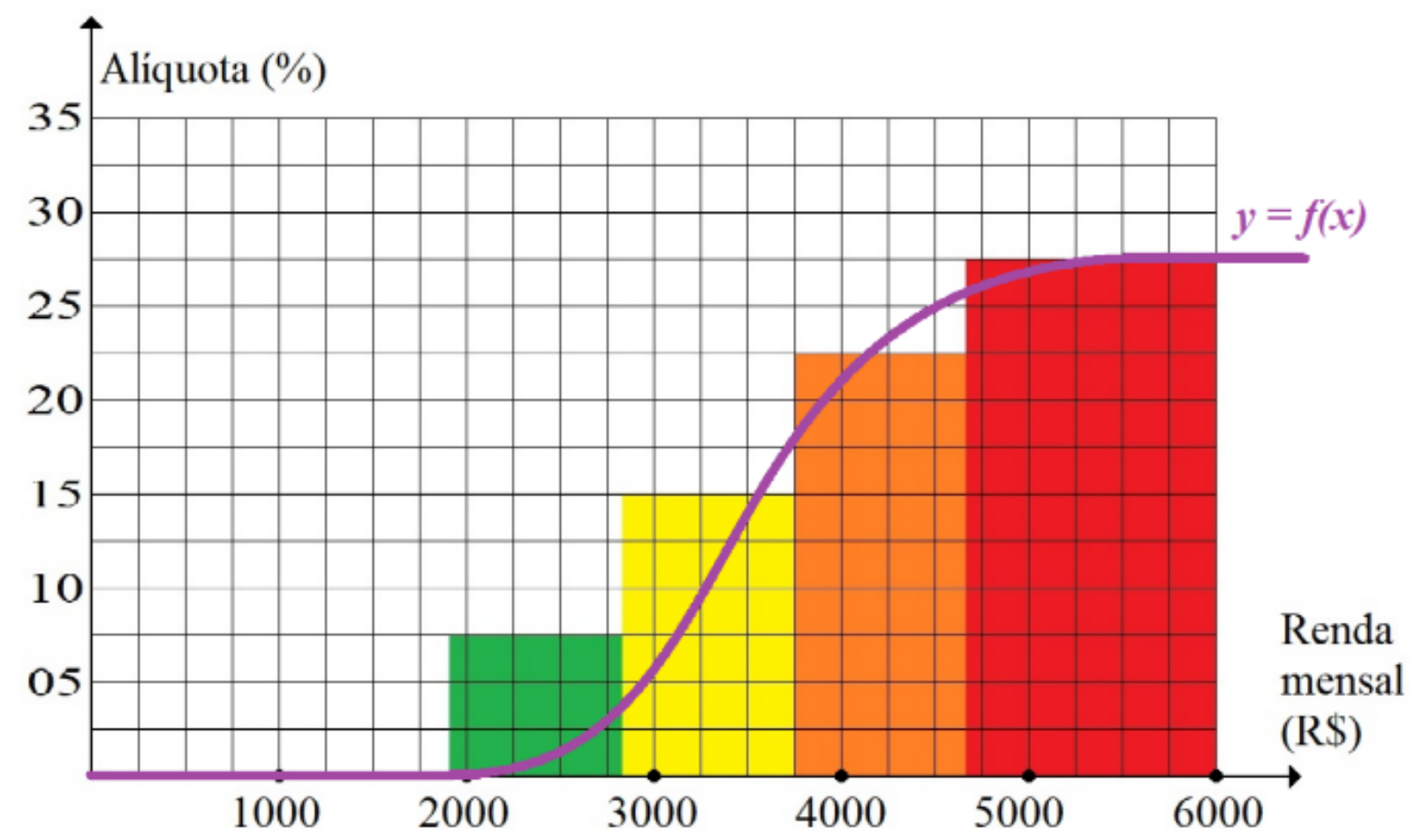

Fonte: elaborado pelos autores.

Através do método "tentativa e erro", chega-se a um exemplo de função que satisfaz as condições (3), (4) e (5), dado pela seguinte expressão: $f(u)=\frac{u^{2}}{u^{2}+1}$ Entretanto, faz-se necessária a introdução de parâmetros (de significado econômico). Pode-se então adotar o formato mais geral: $f(u)=\frac{A u^{2}}{u^{2}+B}$ sendo A,B constantes reais positivas. Tal função deve possuir domínio $+D(f) R \square$ satisfazendo a condição (1).

Para satisfazer a condição (2), basta introduzir uma translação, tomando-se $u=x$ - $R_{\text {min }}$, sendo este novo parâmetro também uma constante real positiva (o significado econômico dos parâmetros será discutido mais adiante). Portanto, o formato final da modelagem matemática proposta é o seguinte:

$$
\mathrm{y}=\mathrm{f}(\mathrm{x})= \begin{cases}0 & \text { se } \mathrm{x} \leq R_{\text {min }} \\ \frac{A\left(x-R_{\text {min }}\right)^{2}}{\left(x-R_{\text {min }}\right)^{2}+B} & \text { se } \mathrm{x}>\mathrm{R}_{\text {min }}\end{cases}
$$

\subsection{Significados Econômicos dos Parâmetros}

Idealmente, o gestor do Imposto de Renda de Pessoa Física - a Receita Federal - precisa definir três parâmetros básicos, a saber:

- $R_{\min }=$ renda mensal até a qual não se cobra imposto (valor atual: $\mathrm{R} \$ 1.903,98$ );

- $R_{\max }=$ renda mensal a partir da qual se cobra alíquota máxima (valor atual: $\mathrm{R} \$ 4.664,69$ );

- $\mathrm{A}=$ alíquota máxima (valor atual: $27,5 \%$ ). 


\subsubsection{Parâmetro $R_{\min }$}

Por definição, $f(x)=0$ para $x \leq R_{\text {min }}$. Logo, o parâmetro matemático corresponde ao parâmetro econômico. Deve-se verificar se a função é contínua no ponto . Para tanto, é suficiente observar que:

$$
\lim _{x \rightarrow R_{\text {min }}} f(x)=\lim _{x \rightarrow R_{\min }} \frac{A\left(x-R_{\min }\right)^{2}}{\left(x-R_{\min }\right)^{2}+B}=\frac{\lim _{x \rightarrow R_{\min }} A(0)^{2}}{(0)^{2}+B}=\frac{0}{B}=0
$$

\subsubsection{Parâmetro A}

O gráfico da função contém uma reta assíntota horizontal, a qual impõe um limite para a alíquota máxima. Deve-se comentar que essa é a principal diferença entre o modelo estratificado e o modelo suave, ambos contínuos. Considere-se $\mathrm{x} \geq \mathrm{R}_{\max }$. No modelo atual, a alíquota máxima é atingida, enquanto que no modelo proposto essa alíquota é um valor limite. O cálculo do limite $\lim _{x \rightarrow+\infty} f(x)$ pode ser feito utilizando-se a regra de L'Hôpital:

$$
\begin{gathered}
f(x)=\frac{\lim _{x \rightarrow+\infty} A\left(x-R_{\text {min }}\right)^{2}}{\left(x-R_{\text {min }}\right)^{2}+B}=\frac{\lim _{x \rightarrow+\infty} 2 A\left(x-R_{\text {min }}\right)^{2}}{2\left(x-R_{\text {min }}\right)}=A \\
\lim _{x \rightarrow+\infty} \square
\end{gathered}
$$

Isso mostra que, para valores suficientemente grandes, a alíquota se aproxima de $A$, que é tomada então como o valor da alíquota máxima. A questão é: quão rápido a função se aproxima desse valor? É o que se discute a seguir.

\subsubsection{Parâmetro B}

Para que o modelo ora apresentado seja efetivo, é imperativo que, para $x$ próximo de $R_{\text {max }}$, o valor de $y=f(x)$ esteja próximo de $A$. Em outras palavras, vamos garantir que se tenha $A-f\left(R_{\max }\right)=\varepsilon$, sendo $\varepsilon$ um valor positivo pequeno (vide Figura 3 ). 
Figura 3 - Busca do significado de B.

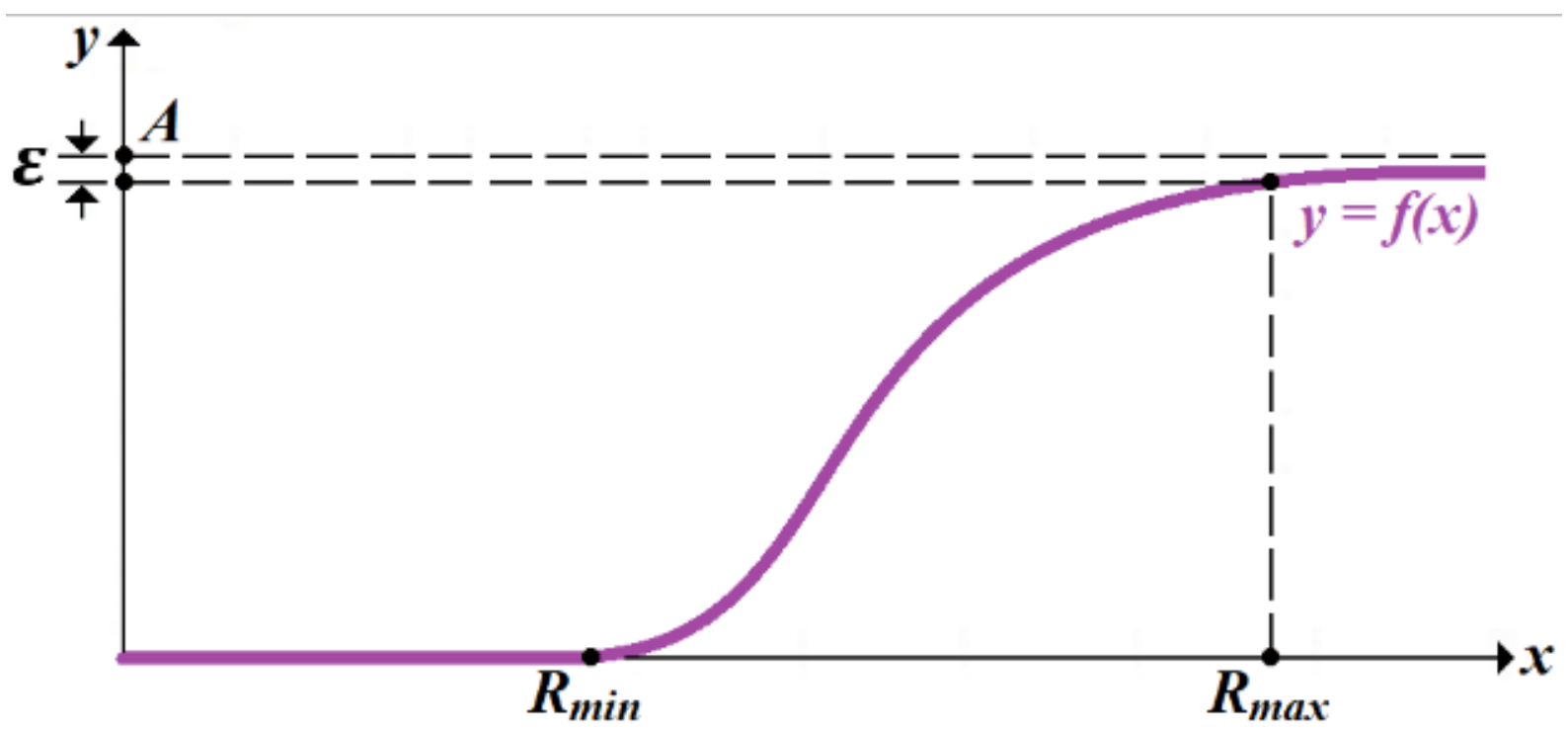

Fonte: elaborado pelos autores.

Pode-se começar escrevendo-se a função da seguinte maneira (para $x>R_{\min }$ ):

$$
y=f(x)=\frac{A\left(x-R_{\text {min }}\right)^{2}}{\left(x-R_{\text {min }}\right)^{2}+B}=A-\frac{A B}{\left(x-R_{\text {min }}\right)^{2}+B} .
$$

Portanto:

$$
\begin{gathered}
A-f\left(R_{\text {max }}\right)=\varepsilon \Rightarrow A-\left[A-\frac{A B}{\left(R_{\text {max }}-R_{\text {min }}\right)^{2}+B}\right]=\varepsilon \Rightarrow \\
\frac{A B}{\left(R_{\text {max }}-R_{\text {min }}\right)^{2}+B}=\varepsilon \Rightarrow A B=\varepsilon\left(R_{\text {max }}-R_{\text {min }}\right)^{2}+\varepsilon B \Rightarrow \\
B(A-\varepsilon)=\varepsilon\left(R_{\text {max }}-R_{\text {min }}\right)^{2} \Rightarrow B=\frac{\varepsilon\left(R_{\text {max }}-R_{\text {min }}\right)^{2}}{A-\varepsilon}
\end{gathered}
$$

Os autores sugerem, por tentativa e erro, adotar-se $\varepsilon=0,01$ ponto percentual. Nesse caso, o gestor pode calcular o parâmetro B pela seguinte fórmula:

$$
B=\frac{0.01\left(R_{\max }-R_{\min }\right)^{2}}{A-0.01}
$$

\subsection{Cálculo do Imposto no Modelo Proposto}

Considere-se $x>R_{\min }$ (isso é, a renda de uma pessoa física que não está isenta do pagamento do IRPF). No modelo estratificado, o cálculo do imposto equivale a uma soma de áreas retangulares, conforme a Figura 4. 


\section{Figura 4 - Modelo estratificado.}

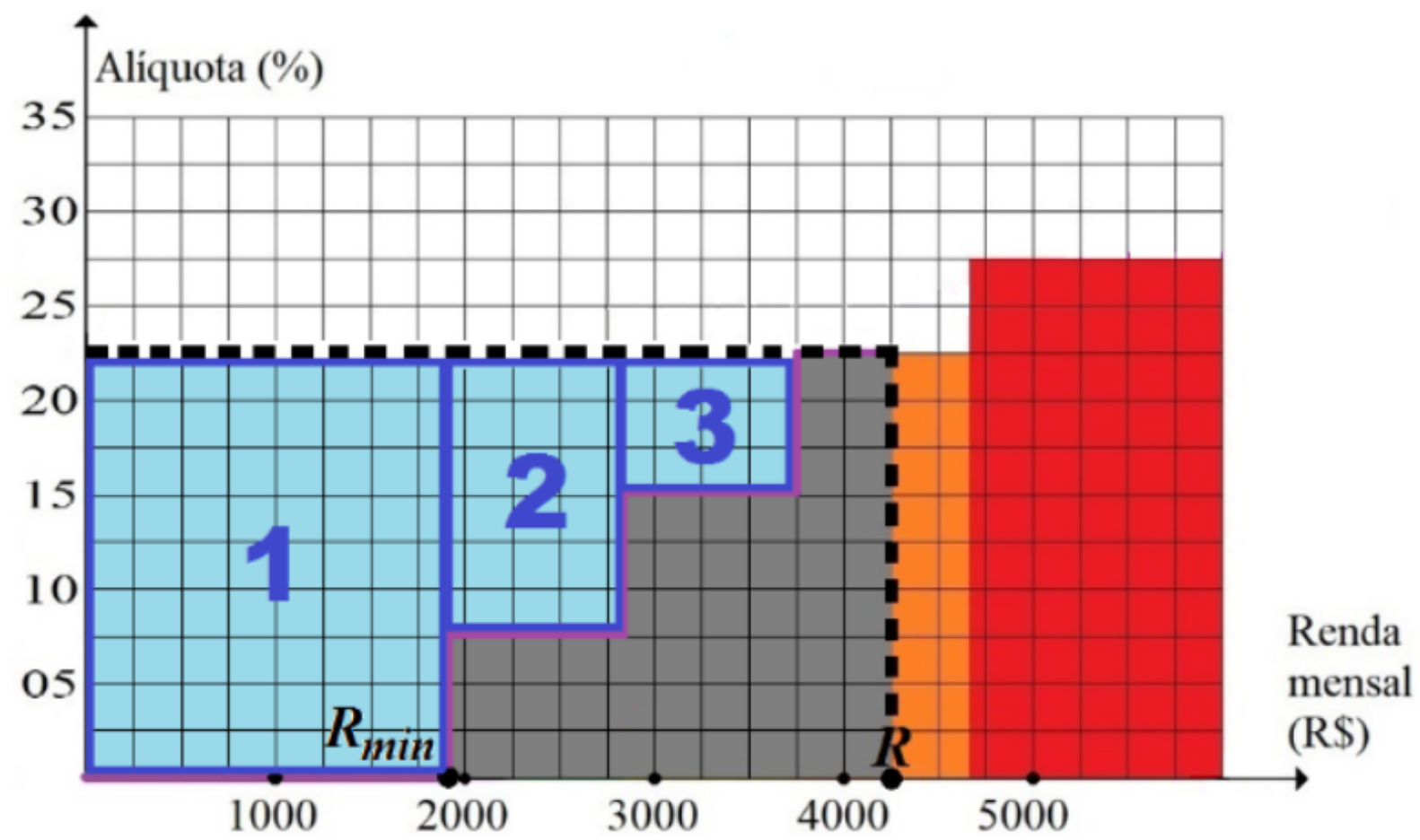

Fonte: elaborado pelos autores.

$\mathrm{Na}$ Figura 4, a pessoa física possui uma renda entre $\mathrm{R} \$ 3.751,06$ e $\mathrm{R} \$ 4.664,68$, cuja alíquota é de $22,5 \%$. Porém, essa alíquota incide apenas sobre o que exceder R $\$ 3.751,06$ (isso é, sobre - $\mathrm{R} \$ 3.751,06)$. Considerando a faixa de $\mathrm{R} \$ 2.826,66$ até $\mathrm{R} \$ 3.751,05$, a alíquota é de $15 \%$ sobre a diferença (isso é, sobre $\mathrm{R} \$ 3.751,06$ - $\mathrm{R} \$ 2.826,66=\mathrm{R} \$ 924,40$ ), enquanto que a faixa de $\mathrm{R} \$ 1.903,99$ até $\mathrm{R} \$ 2.826,65$ gera uma alíquota de 7,5\% sobre a diferença $(\mathrm{R} \$ 922,66)$, conforme a tabela 1. A área apresentada na cor cinza, na Figura 4, corresponde ao valor do imposto devido.

A parcela a deduzir faz uma compensação: valor gerado pela alíquota incidente sobre a renda total menos o valor que teria sido cobrado a mais, ou seja, correspondendo à área em azul claro na Figura 4. Observando-se as áreas 1, 2 e 3 na mesma figura, tem-se:

- Área $1=22,5 \%$ sobre $\mathrm{R} \$ 1.903,98=\mathrm{R} \$ 428,40$

- Área $2=15 \%$ sobre $\mathrm{R} \$ 922,66=\mathrm{R} \$ 138,40$.

- Área $3=7,5 \%$ sobre $\mathrm{R} \$ 924,40=\mathrm{R} \$ 69,33$.

- Soma $=\mathrm{R} \$ 636,13$.

Logo, a parcela a deduzir é de $\mathrm{R} \$ 636,13$, conforme a tabela 1 .

Já no modelo suave, o equivalente é uma integral, como mostra a figura 5. 
Figura 5 - Integral.

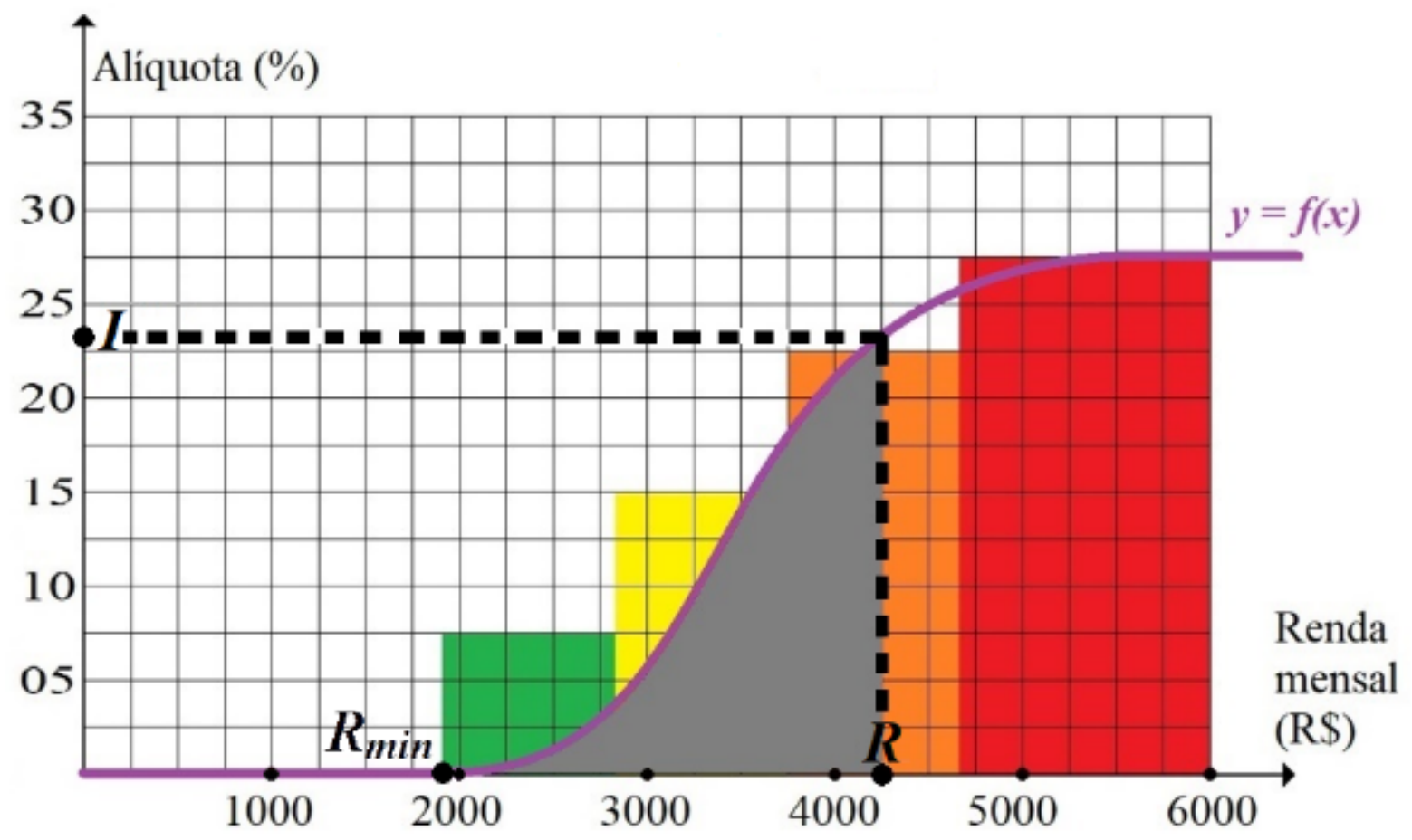

Fonte: elaborado pelos autores.

A integral equivalente ao imposto de uma renda fica:

$$
I=\int_{R}^{R_{\text {min }}} \frac{A\left(x-R_{\text {min }}\right)^{2}}{\left(x-R_{\text {min }}\right)^{2}+B} d x
$$

Pode-se primeiramente calcular a integral indefinida correspondente, utilizando-se a substituição simples (consequentemente):

$$
\begin{gathered}
I=\int \frac{A\left(x-R_{\text {min }}\right)^{2}}{\left(x-R_{\text {min }}\right)^{2}+B} d x=\int \frac{A u^{2}}{u^{2}+B} d u=A \int \frac{u^{2}}{u^{2}+B} d u= \\
A \int\left(1-\frac{B}{u^{2}+B}\right) d u=A \int\left(1-B \cdot \frac{A u^{2}}{u^{2}+B}\right) d u= \\
\text { A. }\left[u-B\left(\frac{1}{\sqrt{B}} \cdot \operatorname{arctg} \frac{u}{\sqrt{B}}\right)\right]=A \cdot\left[u-\sqrt{B} \cdot \operatorname{arctg} \frac{u}{\sqrt{B}}\right]= \\
\text { A. }\left[x-R_{\text {min }}-\sqrt{B} \cdot \operatorname{arctg}\left(\frac{x-R_{\text {min }}}{\sqrt{B}}\right)\right]+\text { constante }
\end{gathered}
$$

Portanto:

$$
\begin{gathered}
I=\left.A \cdot\left[x-R_{\text {min }}-\sqrt{B} \cdot \operatorname{arctg}\left(\frac{x-R_{\text {min }}}{\sqrt{B}}\right)\right]\right|_{R_{\text {min }}} ^{R} \Rightarrow \\
I=A \cdot\left\{\left[R-R_{\text {min }}-\sqrt{B} \cdot \operatorname{arctg}\left(\frac{R-R_{\text {min }}}{\sqrt{B}}\right)\right]-\left[0-\sqrt{B} \cdot \operatorname{arctg}\left(\frac{0}{\sqrt{B}}\right)\right]\right\} \Rightarrow
\end{gathered}
$$




$$
I=A \cdot\left[R-R_{\text {min }}-\sqrt{B} \cdot \operatorname{arctg}\left(\frac{R-R_{\text {min }}}{\sqrt{B}}\right)\right]
$$

\section{RESULTADOS}

\subsection{Comparação entre os Dois Métodos para Cálculo do Imposto}

A fim de automatizar os cálculos, foi criado um programa em linguagem $\mathrm{C}++$, apresentado no apêndice, que gera automaticamente uma tabela para algumas faixas de valores de renda mensal. Cada linha de saída apresenta quatro valores:

- a renda mensal;

- o imposto E calculado pelo método atual;

- o imposto S calculado pelo novo método;

- a razão $\mathrm{S} / \mathrm{E}$ em porcentagem.

Utilizando-se esse programa, obtém-se a Tabela 2 de comparação. 
Tabela 2 - Imposto calculado pelos dois métodos: atual e proposto.

\begin{tabular}{|c|c|c|c|}
\hline Renda mensa (R\$) & $\begin{array}{c}\mathrm{E}=\text { Imposto }(\mathrm{R} \$) \\
\text { (modelo estratificado ) }\end{array}$ & $\begin{array}{c}\mathrm{S}=\text { Integral }(\mathrm{R} \$) \\
\text { (modelo suave) }\end{array}$ & Razão S/E (\%) \\
\hline $1.500,00$ & 0,00 & 0,00 & - \\
\hline $2.000,00$ & 7,20 & 0,02 & 0,26 \\
\hline $2.500,00$ & 44,70 & 4,25 & 9,51 \\
\hline $3.000,00$ & 95,20 & 23,87 & 25,07 \\
\hline $3.500,00$ & 170,20 & 64,21 & 37,73 \\
\hline $4.000,00$ & 263,87 & 124,42 & 47,15 \\
\hline $4.500,00$ & 376,37 & 201,02 & 53,41 \\
\hline $5.000,00$ & 505,64 & 290,31 & 57,41 \\
\hline $5.500,00$ & 643,14 & 389,20 & 60,52 \\
\hline $6.000,00$ & 780,64 & 495,36 & 63,46 \\
\hline $6.500,00$ & 918,14 & 607,04 & 66,12 \\
\hline $7.000,00$ & $1.055,64$ & 722,99 & 68,49 \\
\hline $7.500,00$ & $1.193,14$ & 842,28 & 70,59 \\
\hline $8.000,00$ & $1.330,64$ & 964,22 & 72,46 \\
\hline $8.500,00$ & $1.468,14$ & $1.088,28$ & 74,13 \\
\hline $9.000,00$ & $1.605,64$ & $1.214,08$ & 75,61 \\
\hline $9.500,00$ & $1.743,14$ & $1.341,30$ & 76,95 \\
\hline $10.000,00$ & $1.880,64$ & $1.469,71$ & 78,15 \\
\hline $20.000,00$ & $4.630,64$ & $4.140,82$ & 89,42 \\
\hline $30.000,00$ & $7.380,64$ & $6.867,48$ & 93,05 \\
\hline $40.000,00$ & $10.130,64$ & $9.606,34$ & 94,82 \\
\hline $50.000,00$ & $12.880,64$ & $12.349,82$ & 95,88 \\
\hline $60.000,00$ & $15.630,64$ & $15.095,54$ & 96,58 \\
\hline $70.000,00$ & $18.380,64$ & $17.842,51$ & 97,07 \\
\hline $80.000,00$ & $21.130,64$ & $20.590,26$ & 97,44 \\
\hline $90.000,00$ & $23.880,64$ & $23.338,52$ & 97,73 \\
\hline $100.000,00$ & $26.630,64$ & $26.087,14$ & 97,96 \\
\hline
\end{tabular}

Fonte: formatado pelos autores a partir da saída do Programa 1.

A tabela permite concluir que:

- o imposto de renda é sempre menor pelo modelo suave;

- à medida que a renda aumenta, ambos tendem a se igualar

O modelo suave é mais equitativo. Na tabela tradicional do imposto de renda, uma grande faixa de pessoas físicas é direcionada para uma mesma alíquota. Na nova proposta, a alíquota é "customizada" - cada renda está em correspondência biunívoca com sua alíquota. Além disso, conforme mostrado acima, um bom ajuste do valor de EPSILON (e, consequentemente, do parâmetro B) faz com que o imposto final seja menor do que no modelo atual. 


\section{CONCLUSÃO}

Por tudo quanto foi apresentado anteriormente, percebe-se que a substituição do modelo estratificado de cálculo do IRPF pelo modelo suave é notoriamente mais equitativa, especialmente para as pessoas físicas que estão alocadas nas faixas das alíquotas menores.

Além disso, o modelo proposto poderá diminuir a sonegação. De acordo com dados da Rede Brasil Atual ${ }^{3}$ e da Carta $\mathrm{Capital}^{4}$, sonegam-se cerca de $\mathrm{R} \$ 500$ bilhões anuais - tornando o Brasil vice-líder mundial da sonegação. Desse total, o maior montante sonegado é da dívida com o INSS - Instituto Nacional do Seguro Social, seguido da dívida de ICMS - Imposto sobre Operações relativas à Circulação de Mercadorias e Prestação de Serviços e do Imposto de Renda. Ainda de acordo com a Carta Capital, a perda por sonegação é sete vezes maior do que a perda por corrupção (o Brasil é o $69^{\circ}$ país menos corrupto do mundo). A desigualdade da tributação está intimamente associada à desigualdade de renda.

Um modelo mais equitativo poderá servir de incentivo ao contribuinte para pagar o Imposto de Renda Pessoa Física. Principalmente se o montante arrecadado for realmente convertido em benefícios para a população, como educação, saúde, infraestrutura, programas sociais, cultura, esporte, salários do funcionalismo público federal etc.

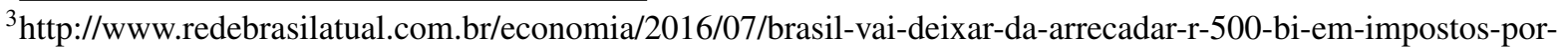
sonegacao-6775.html

${ }^{4}$ https://www.cartacapital.com.br/economia/a-sonegacao-fiscal-destroi-o-brasil
} 


\section{REFERÊNCIAS}

90 anos de Imposto de Renda no Brasil: de Gulliver ao Leão. Disponível em: <http://www. estadao.com.br/infograficos/economia,90-anos-de-imposto-de-renda-no-brasil-de-gulliver-aoleao,208636>. Acesso em: 12 jun. 2018.

A desigualdade de renda e de tributação no Brasil em 3 gráficos. Disponível em: $<$ https://www.nexojornal.com.br/expresso/2016/12/21/A-desigualdade-de-renda-e-detributa\%C3\%A7\%C3\%A3o-no-Brasil-em-3-gr\%C3\%A1ficos>. Acesso em: 28 mai. 2018.

A sonegação fiscal destrói o Brasil. Disponível em: <https://www.cartacapital.com.br/economia/ a-sonegacao-fiscal-destroi-o-brasil>. Acesso em: 12 jun. 2018.

Brasil vai deixar de arrecadar R $\$ 500$ bi em 2016 devido à sonegação de impostos. Disponível em: <http://www.redebrasilatual.com.br/economia/2016/07/brasil-vai-deixar-da-arrecadarr-500-bi-em-impostos-por-sonegacao-6775.html>. Acesso em: 12 jun. 2018.

Lei $\mathrm{N}^{0}$ 13.149, de 21 de julho de 2015. Disponível em: <http://www.planalto.gov.br/ccivil_03/ _ato2015-2018/2015/lei/l13149.htm>. Acesso em: 28 mai. 2018.

Medida Provisória No 670, de 10 de março de 2015. Disponível em: <http://www.planalto.gov. br/ccivil_03/_ato2015-2018/2015/mpv/mpv670.htm>. Acesso em: 28 mai. 2018.

Não correção da tabela do Imposto de Renda é absurdo, critica Salete Lemos. Disponível em: http://www.redetv.uol.com.br/jornalismo/redetvnews/videos/economia/nao-correcaoda-tabela-do-imposto-de-renda-e-absurdo-critica-salete-lemos. Acesso em: 01 jun. 2018.

Não corrigir a tabela do IR é aumento de imposto. Disponível em: <http://www.fepesp.org.br/ geral/noticias/tabela-do-imposto-de-renda-tem-que-ser-corrigida>. Acesso em: 01 jun. 2018.

REIS, Thiago L. B. Integral definida: conteúdos de ensino e estratégias de aprendizagem. 2015. Dissertação de Mestrado — Ensino de Ciências e Matemática na PUC MINAS, 244p.

STEWART, James. Cálculo - Volume 1. 6. ed. São Paulo: Cengage Learning, 2011. 535p. ISBN 978-85-221-0660-8.

Saiba para onde vai o dinheiro arrecadado com o imposto de renda. Disponível em: <http://www.ebc.com.br/noticias/economia/2013/02/verbas-arrecadadas-com-imposto-derendas-tem-destinacoes-diversas>. Acesso em: 12 jun. 2018.

Sonegação de impostos é sete vezes maior que a corrupção. Disponível em: <https://www.car tacapital.com.br/economia/sonegacao-de-impostos-e-sete-vezes-maior-que-a-corrupcao9109.html>. Acesso em: 12 jun. 2018.

Tabela do Imposto de Renda tem que ser corrigida. Disponível em: <http://www.fepesp.org.br/ geral/noticias/tabela-do-imposto-de-renda-tem-que-ser-corrigida>. Acesso em: 28 mai. 2018. 


\section{APÊNDICE}

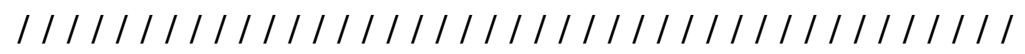

// Calculo de IRPF segundo duas vertentes

// Prof. Thiago Linhares Brant Reis

$/ /$ Prof. Alexandre Duarte Washington

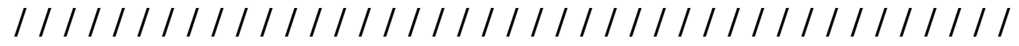

\#include <iostream $>$

\#include $\langle$ cstdlib $>$

\#include <cmath>

\#include <iomanip >

using namespace std;

// renda mensal at a qual $n$ o se cobra imposto const double RMIN = 1903.98;

// renda mensal a partir da qual se cobra al quota m xima const double RMAX = 4664.69;

// aliquota maxima

const double $\mathrm{A}=27.5$;

// outras faixas de renda e respectivas aliquotas

const double $\mathrm{R} 1=2826.65$

const double A1 $=7.5$;

const double $\mathrm{R} 2=3751.05$

const double $\mathrm{A} 2=15.0 ;$

const double R3 = 4664.68;

const double $\mathrm{A} 3=22.5$

// deducoes

const double D1 = 142.80 ;

const double D2 = 354.80;

const double D3 = 636.13;

const double DMAX $=869.36$;

// rendas diversas

const int QUANTIDADE_1 $=18$;

const int QUANTIDADE_2 $=9$;

const int QUANTIDADE_TOTAL = QUANTIDADE_1+QUANTIDADE_2;

const double DELTA_1 = 500.0;

const double RENDA_INICIAL_1 $=1500.0$;

const double RENDA_FINAL_1 $=10000.0$;

const double DELTA_2 $=10000.0$;

const double RENDA_INICIAL_2 = RENDA_FINAL_1+DELTA_2;

const double RENDA_FINAL_2 $=1000000.0$;

// outros

const double EPSILON $=0.1$;

double valorDoImposto(double renda) 


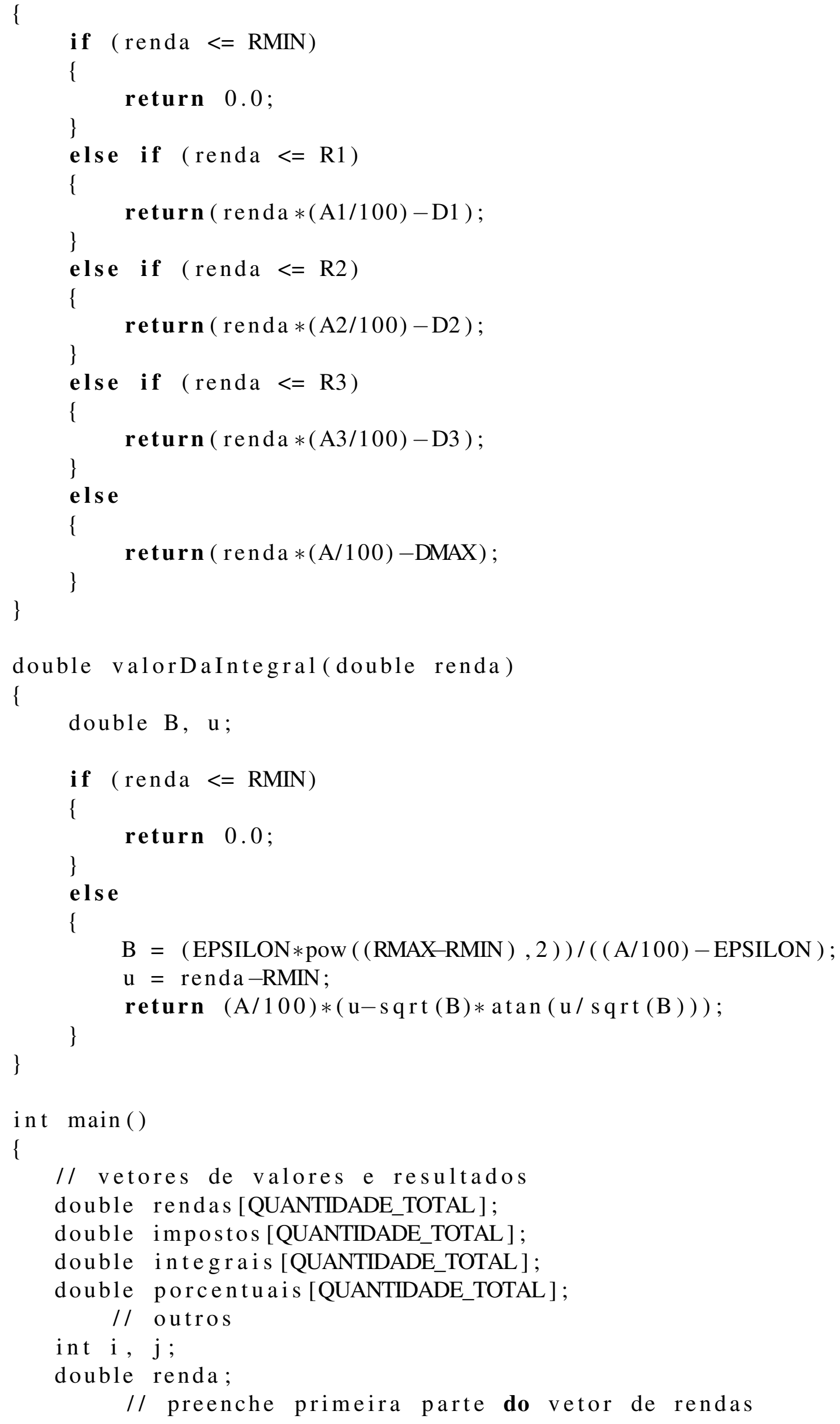




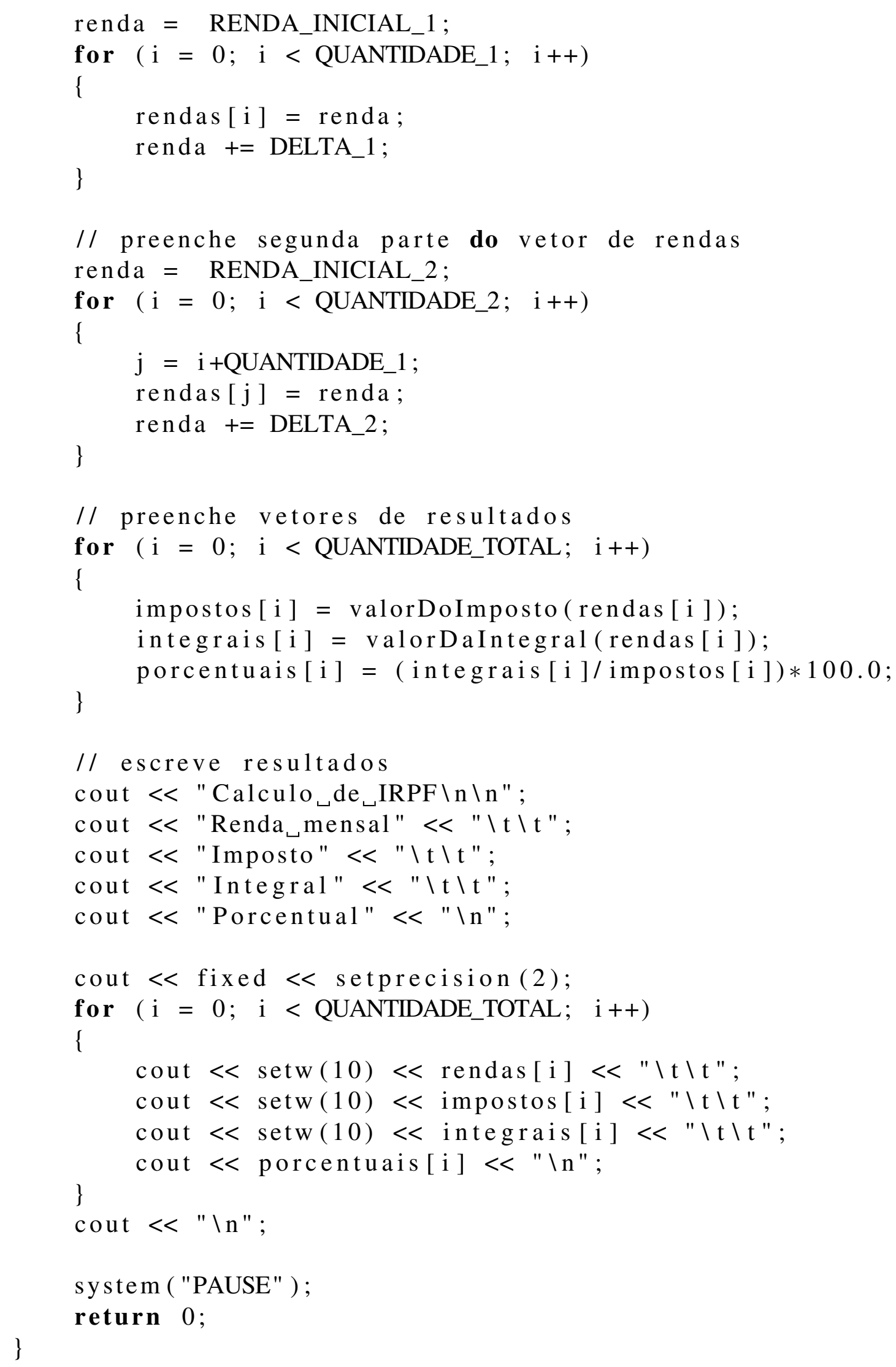

